\title{
ON STRONGLY CLEAN MATRIX RINGS
}

\author{
LINGLING FAN \\ Department of Mathematics and Statistics, Memorial University of Newfoundland, \\ St.John's, A1C 5S7, Canada \\ e-mail:lingling@math.mun.ca \\ and XIANDE YANG ${ }^{1}$ \\ College of Computer Science, Wuhan Institute of Technology, Wuhan, 430073 P.R. China \\ e-mail: xiande@math.mun.ca
}

(Received 17 March, 2006; revised 3 July, 2006: accepted 31 July, 2006)

\begin{abstract}
A ring $R$ with identity is called strongly clean if every element of $R$ is the sum of an idempotent and a unit that commute. For a commutative local ring $R, n=3,4$, and $m, k, s \in \mathbb{N}$ it is proved that $\mathbb{M}_{n}(R)$ is strongly clean if and only if $\mathbb{M}_{n}(R[[x]])$ is strongly clean if and only if $\mathbb{M}_{n}\left(R\left[\left[x_{1}, x_{2}, \ldots, x_{m}\right]\right]\right)$ is strongly clean if and only if $\mathbb{M}_{n}\left(\frac{R[x]}{\left(x^{k}\right)}\right)$ is strongly clean if and only if $\mathbb{M}_{n}\left(\frac{R\left[x_{1}, x_{2}, \ldots, x_{s}\right]}{\left(x_{1}^{n_{1}}, x_{2}^{n_{2}}, \ldots, x_{s}^{n_{s}}\right)}\right)$ is strongly clean if and only if $\mathbb{M}_{n}(R \propto R)$ is strongly clean where $R \propto R=\left\{\left(\begin{array}{ll}a & b \\ 0 & a\end{array}\right): a, b \in R\right\}$ is the trivial extension of $R$. This extends a result of J. Chen, X. Yang and Y. Zhou [5] from $n=2$ to 3 and 4 .
\end{abstract}

2000 Mathematics Subject Classification. Primary 16U99, 16S50, 16S99. Secondary 16U60, 16U10.

1. Introduction. In this paper, $R$ is an associative ring with identity. A ring $R$ is called clean if for every element $a \in R$, there exist an idempotent $e$ and a unit $u$ in $R$ such that $a=e+u$ [10], and $R$ is called strongly clean if in addition $e u=u e[\mathbf{1 1}]$. By Han and Nicholson [8], the cleanness of the ring $R$ implies that of the matrix ring $\mathbb{M}_{n}(R)$ for any $n \geq 1$. But if $R$ is strongly clean, the matrix ring $\mathbb{M}_{n}(R)$ with $n>1$ may not be strongly clean. For example, the matrix ring $\mathbb{M}_{2}\left(\mathbb{Z}_{(2)}\right)$ is not strongly clean. This fact was observed by Sánchez Campos [12] and by Wang and Chen [13] independently (answering two questions of Nicholson in [11]). When is the matrix ring over a strongly clean ring still strongly clean? Recently, the authors found an equation condition [5, Theorem 8] for $\mathbb{M}_{2}(R)$ over a commutative local ring to be strongly clean. In [4], the authors defined $n-S R C$ ring (see Definition 2.1) and found the matrix ring $\mathbb{M}_{n}(R)$ over a commutative local ring is strongly clean if and only if $R$ is $n-S R C$.

Let $R[[x]]$ denote the formal power series ring with elements of the form $\sum_{i=0}^{\infty} r_{i} x^{i}$, $r_{i} \in R, x^{0}=1$. In [5, Theorem 9] it is proved that $\mathbb{M}_{2}(R)$ over a commutative local ring $R$ is strongly clean if and only if $\mathbb{M}_{2}(R[[x]])$ is strongly clean. This is equivalent to saying that if $\mathbb{M}_{2}(R)$ is strongly clean, then the power series extension $\left(\mathbb{M}_{2}(R)\right)[[x]]$ $\left(\cong \mathbb{M}_{2}(R[[x]])\right)$ is also strongly clean. However, it is not known, whether or not $R[[x]]$ is also strongly clean wherever $R$ is a strongly clear ring.

${ }^{1}$ Correspondence: Xiande Yang, Department of Mathematics and Statistics, Memorial University of Newfoundland, St. John's, A1C 5S7, Canada. Fax:(709)737-3010. 
Generally, if $\mathbb{M}_{2}(R)$ has a property, one may think $\mathbb{M}_{n}(R)$ also has the property. However, in [4], the authors gave an example showing that the strong cleanness of $\mathbb{M}_{2}(R)$ over a commutative local ring $R$ need not imply the strong cleanness of $\mathbb{M}_{3}(R)$. Hence, the equivalence of strong cleanness for $\mathbb{M}_{2}(R)$ and $\left(\mathbb{M}_{2}(R)\right)[[x]]$ need not imply the equivalence of strong cleanness for $\mathbb{M}_{n}(R)$ and $\left(\mathbb{M}_{n}(R)\right)[[x]]\left(\cong \mathbb{M}_{n}(R[[x]])\right)$.

Here we have proved the following main result.

THEOREM. Let $R$ be a commutative local ring, and let $n=3,4$, and $m, k, s \in \mathbb{N}$. Then the following are equivalent (see Definition 2.8 for $R \propto R$ ).

(1) $\mathbb{M}_{n}(R)$ is strongly clean.

(2) $\mathbb{M}_{n}(R[[x]])$ is strongly clean.

(3) $\mathbb{M}_{n}\left(\frac{R[x]}{\left(x^{m}\right)}\right)$ is strongly clean.

(4) $\mathbb{M}_{n}\left(R\left[\left[x_{1}, x_{2}, \ldots, x_{k}\right]\right]\right)$ is strongly clean.

(5) $\mathbb{M}_{n}\left(\frac{R\left[x_{1}, x_{2}, \ldots, x_{s}\right]}{\left(x_{1}^{n_{1}}, x_{2}^{n_{2}}, \ldots, n_{s}^{n_{S}}\right)}\right)$ is strongly clean.

(6) $\mathbb{M}_{n}(R \propto R)$ is strongly clean.

As usual, we use $U(R)$ and $J(R)$ to denote the group of units and the Jacobson radical of $R$ respectively. For a field $F$, if $h(t), g(t) \in F[t]$, then $\operatorname{gcd}(h(t), g(t))$ denotes the monic greatest common divisor of polynomials $h(t), g(t)$.

\section{Main results.}

Definition 2.1. [4] Let $R$ be a commutative local ring. In $R[t]$, a factorization $h(t)=h_{0}(t) h_{1}(t)$ of a monic polynomial $h(t)$ is said to be an $S R C$ factorization if $h_{0}(0), h_{1}(1)$ are units and $\overline{h_{0}}(t), \overline{h_{1}}(t)$ are coprime in the principal ideal domain $\bar{R}[t]$ $(=R / J(R)[t]) . R$ is an $S R C$ ring ( resp. $n-S R C$ ring) if every monic polynomial (resp. every monic polynomial of degree $n$ ) has an $S R C$ factorization.

LEMMA 2.2. [4] Let $R$ be a commutative local ring. Then $R$ is $n-S R C$ if and only if $\mathbb{M}_{n}(R)$ is strongly clean; $R$ is $S R C$ if and only if $\mathbb{M}_{n}(R)$ is strongly clean for all $n \in \mathbb{N}$.

THEOREM 2.3. Let $R$ be a commutative local ring. Then the following are equivalent:

(1) $R$ is a 3-SRC ring.

(2) $R[[x]]$ is a 3-SRC ring.

Proof. $(1) \Rightarrow(2): R[[x]]$ is a commutative local ring with $J(R[[x]])=J(R)+x R[[x]]$. Define $\theta: R[[x]] \rightarrow R$ by $\theta\left(r_{0}+r_{1} x+r_{2} x^{2}+\cdots\right)=r_{0}$. It is easy to verify that $\theta$ is an epimorphism. Let $\eta_{J(R)}: R \rightarrow R / J(R)$ be the natural ring epimorphism with $\eta_{J(R)}(r)=$ $\bar{r}=r+J(R)$ and $\eta_{J(R[x]])}$ be defined similarly. Then the following diagram commutes where $\bar{\theta}(r+J(R[[x]]))=\theta(r)+J(R)=r+J(R)=\bar{r}, r \in R$, is an isomorphism since it is a field epimorphism.

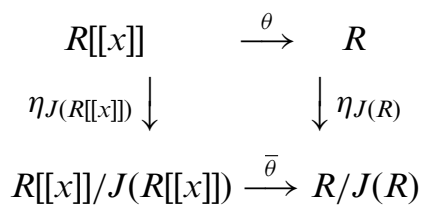

Further it induces the following commutative diagram where $\eta_{J(R)}^{\prime}\left(r_{0}+r_{1} t+\cdots+\right.$ $\left.r_{n} t^{n}\right)=\eta_{J(R)}\left(r_{0}\right)+\eta_{J(R)}\left(r_{1}\right) t+\cdots+\eta_{J(R)}\left(r_{n}\right) t^{n} \quad$ with $\quad r_{0}+r_{1} t+\cdots+r_{n} t^{n} \in R[t] \quad$ and 
$\eta_{J(R[[x])}^{\prime}$ defined similarly. $\theta^{\prime}\left(f_{0}+f_{1} t+\cdots+f_{n} t^{n}\right)=\theta\left(f_{0}\right)+\theta\left(f_{1}\right) t+\cdots+\theta\left(f_{n}\right) t^{n}$ with $f_{0}+f_{1} t+\cdots+f_{n} t^{n} \in R[[x]][t], \quad \bar{\theta}^{\prime}\left(\overline{f_{0}}+\overline{f_{1}} t+\ldots+\overline{f_{n}} t^{n}\right)=\bar{\theta}\left(\overline{f_{0}}\right)+\bar{\theta}\left(\overline{f_{1}}\right) t+\cdots+\bar{\theta}\left(\overline{f_{n}}\right) t^{n}$ with $\overline{f_{0}}+\overline{f_{1}} t+\ldots+\overline{f_{n}} t^{n} \in \frac{R[[x]]}{J(R[x]])}[t]$ and $\bar{\theta}^{\prime}$ is an isomorphism.

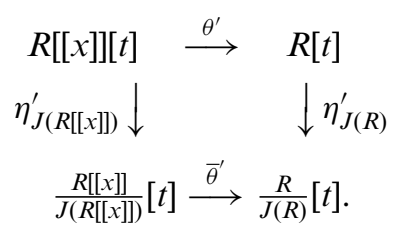

Let $h(t)=f_{0}+f_{1} t+f_{2} t^{2}+t^{3} \in R[[x]][t]$ with $f_{i}=r_{i 0}+r_{i 1} x+r_{i 2} x^{2}+\cdots, i=0,1,2$.

I: If $h(0) \in U(R)$, then let $h_{0}(t)=h(t), h_{1}(t)=1$ and if $h(1) \in U(R)$, then let $h_{0}(t)=1$, $h_{1}(t)=h(t)$. In either case, $h(t)$ has an $S R C$ factorization.

II: If $h(0)=f_{0} \in J(R[[x]]), h(1)=f_{0}+f_{1}+f_{2}+1 \in J(R[[x]])$, i.e., $r_{00} \in J(R)$, and $r_{00}+$ $r_{10}+r_{20}+1 \in J(R)$, we want to prove $h(t)$ still has an $S R C$ factorization.

Let $h^{\prime}(t)=\theta^{\prime}(h(t))$. Then $h^{\prime}(t)=r_{00}+r_{10} t+r_{20} t^{2}+t^{3}, h^{\prime}(0)=r_{00} \in J(R)$ and $h^{\prime}(1)=$ $r_{00}+r_{10}+r_{20}+1 \in J(R)$. Since $R$ is a $3-S R C$ ring, there exist

$$
\text { (a) }\left\{\begin{array}{l}
h_{0}^{\prime}(t)=a_{00}+a_{10} t+t^{2} \\
h_{1}^{\prime}(t)=b_{00}+t
\end{array}\right.
$$

or

$$
\text { (b) }\left\{\begin{array}{l}
h_{0}^{\prime}(t)=a_{00}+t \\
h_{1}^{\prime}(t)=b_{00}+b_{10} t+t^{2}
\end{array}\right.
$$

such that $h_{0}^{\prime}(0) \in U(R), h_{1}^{\prime}(1) \in U(R), \operatorname{gcd}\left(\eta_{J(R)}^{\prime}\left(h_{0}(t)\right), \eta_{J(R)}^{\prime}\left(h_{1}(t)\right)\right)=1$ and $h^{\prime}(t)=$ $h_{0}^{\prime}(t) h_{1}^{\prime}(t)$.

Case (a):

$$
\left\{\begin{array}{l}
h_{0}^{\prime}(t)=a_{00}+a_{10} t+t^{2} \\
h_{1}^{\prime}(t)=b_{00}+t
\end{array}\right.
$$

Let $h_{0}(t)=A_{0}+A_{1} t+t^{2} \in R[[x]][t]$ with $A_{i}=a_{i 0}+a_{i 1} x+a_{i 2} x^{2}+\cdots, i=0,1$ and $h_{1}(t)=B_{0}+t \in R[[x]]$ with $B_{0}=b_{00}+b_{01} x+b_{02} x^{2}+\cdots$. We prove there exist $A_{0}, A_{1}$ and $B_{0} \in R[[x]]$ such that $h(t)=h_{0}(t) h_{1}(t)$.

$$
\begin{aligned}
h(t)= & h_{0}(t) h_{1}(t) \Leftrightarrow h(t)=f_{0}+f_{1} t+f_{2} t^{2}+t^{3}=\left(A_{0}+A_{1} t+t^{2}\right)\left(B_{0}+t\right) \\
\Leftrightarrow & \left(r_{00}+r_{01} x+r_{02} x^{2}+\cdots\right)+\left(r_{10}+r_{11} x+r_{12} x^{2}+\cdots\right) t \\
& \quad+\left(r_{20}+r_{21} x+r_{22} x^{2}+\cdots\right) t^{2}+t^{3} \\
= & {\left[\left(a_{00}+a_{01} x+a_{02} x^{2}+\cdots\right)+\left(a_{10}+a_{11} x+a_{12} x^{2}+\cdots\right) t+t^{2}\right] } \\
& \times\left[\left(b_{00}+b_{01} x+b_{02} x^{2}+\cdots\right)+t\right] \\
\Leftrightarrow & \left(r_{00}+r_{10} t+r_{20} t^{2}+t^{3}\right)+\left(r_{01}+r_{11} t+r_{21} t^{2}\right) x+\left(r_{02}+r_{12} t+r_{22} t^{2}\right) x^{2}+\cdots \\
= & {\left[\left(a_{00}+a_{10} t+t^{2}\right)+\left(a_{01}+a_{11} t\right) x+\left(a_{02}+a_{12} t\right) x^{2}+\cdots\right] } \\
& \times\left[\left(b_{00}+t\right)+b_{01} x+b_{02} x^{2}+\cdots\right]
\end{aligned}
$$


$\Leftrightarrow$ the following equation system with $a_{i j}$ and $b_{m n}$ as variables is solvable:

$$
\begin{array}{ll}
\left(P_{0}\right): & \left(a_{00}+a_{10} t+t^{2}\right)\left(b_{00}+t\right)=r_{00}+r_{10} t+r_{20} t^{2}+t^{3} \\
\left(P_{n}\right)(n \in \mathbb{N}): & \left(a_{00}+a_{10} t+t^{2}\right) b_{0 n}+\left(a_{01}+a_{11} t\right) b_{0, n-1}+\cdots+\left(a_{0, n-1}+a_{1, n-1} t\right) b_{01} \\
& +\left(a_{0 n}+a_{1 n} t\right)\left(b_{00}+t\right)=r_{0 n}+r_{1 n} t+r_{2 n} t^{2} .
\end{array}
$$

Use mathematical induction on $n:\left(P_{0}\right)$ holds by the assumption of $h_{0}(t)$ and $h_{1}(t)$. Suppose $\left(P_{n}\right), n=0,1, \ldots, k-1$, are solvable; we prove $\left(P_{k}\right)$ is solvable.

$$
\begin{aligned}
\left(P_{k}\right): \quad & \left(a_{00}+a_{10} t+t^{2}\right) b_{0 k}+\left(a_{01}+a_{11} t\right) b_{0, k-1}+\cdots+\left(a_{0, k-1}+a_{1, k-1} t\right) b_{01} \\
& +\left(a_{0 k}+a_{1 k} t\right)\left(b_{00}+t\right)=r_{0 k}+r_{1 k} t+r_{2 k} t^{2} .
\end{aligned}
$$

Since the coefficients of $t$ in $\left(a_{01}+a_{11} t\right) b_{0, k-1}+\cdots+\left(a_{0, k-1}+a_{1, k-1} t\right) b_{01}$ are all known by induction hypothesis, $\left(P_{k}\right)$ is transformed into

$(*): \quad\left(a_{00}+a_{10} t+t^{2}\right) b_{0 k}+\left(a_{0 k}+a_{1 k} t\right)\left(b_{00}+t\right)$

$$
=-\left[\left(a_{01}+a_{11} t\right) b_{0, k-1}+\cdots+\left(a_{0, k-1}+a_{1, k-1} t\right) b_{01}\right]+r_{0 k}+r_{1 k} t+r_{2 k} t^{2} \text {. }
$$

In the equation $(*), a_{0 k}, a_{1 k}$ and $b_{0 k}$ are variables. Transfer $(*)$ into linear equation system:

$$
\left\{\begin{array}{l}
a_{00} b_{0 k}+a_{0 k} b_{00}=r_{0 k}^{\prime} \\
a_{10} b_{0 k}+a_{0 k}+a_{1 k} b_{00}=r_{1 k}^{\prime} \\
b_{0 k}+a_{1 k}=r_{2 k}^{\prime}
\end{array}\right.
$$

with $r_{i k}^{\prime}, i=0,1,2$, being the coefficients of the polynomial on the right hand side of the equation (*). Define matrices $A, X, B$ as following:

$$
A=\left[\begin{array}{ccc}
b_{00} & 0 & a_{00} \\
1 & b_{00} & a_{10} \\
0 & 1 & 1
\end{array}\right], \quad X=\left[\begin{array}{c}
a_{0 k} \\
a_{1 k} \\
b_{0 k}
\end{array}\right], \quad B=\left[\begin{array}{c}
r_{0 k}^{\prime} \\
r_{1 k}^{\prime} \\
r_{2 k}^{\prime}
\end{array}\right] .
$$

Then the above equation can be transformed into the matrix equation $A X=B$. By $h_{0}^{\prime}(0) \in U(R), h_{1}^{\prime}(1) \in U(R), h^{\prime}(0)=h_{0}^{\prime}(0) h_{1}^{\prime}(0) \in J(R)$ and $h^{\prime}(1)=h_{0}^{\prime}(1) h_{1}^{\prime}(1) \in J(R)$ we get $a_{00} \in U(R)$ and $b_{00} \in J(R)$. So $\operatorname{det} A=\left(b_{00}\right)^{2}+a_{00}-a_{10} b_{00}=a_{00}+b_{00}\left(b_{00}-\right.$ $\left.a_{10}\right) \in U(R)$. Hence the matrix equation has a solution, i.e., $\left(P_{k}\right)$ is solvable. So $h_{0}(t)$ and $h_{1}(t)$ exist by induction.

Case (b):

$$
\left\{\begin{array}{l}
h_{0}^{\prime}(t)=a_{00}+t \\
h_{1}^{\prime}(t)=b_{00}+b_{10} t+t^{2}
\end{array}\right.
$$

Let $h_{0}(t)=A_{0}+t \in R[[x]]$ with $A_{0}=a_{00}+a_{01} x+a_{02} x^{2}+\cdots \in R[[x]][t]$ and $h_{1}(t)=$ $B_{0}+B_{1} t+t^{2} \in R[[x]][t]$ with $B_{i}=b_{i 0}+b_{i 1} x+b_{i 2} x^{2}+\cdots, i=0,1$. Now we show there exist $A_{0}, B_{0}$ and $B_{1} \in R[[x]]$ such that $h(t)=h_{0}(t) h_{1}(t)$.

$$
\begin{aligned}
h(t)= & h_{0}(t) h_{1}(t) \Leftrightarrow \\
h(t)= & f_{0}+f_{1} t+f_{2} t^{2}+t^{3}=\left(A_{0}+t\right)\left(B_{0}+B_{1} t+t^{2}\right) \\
\Leftrightarrow & \left(r_{00}+r_{01} x+r_{02} x^{2}+\cdots\right)+\left(r_{10}+r_{11} x+r_{12} x^{2}+\cdots\right) t \\
& \quad+\left(r_{20}+r_{21} x+r_{22} x^{2}+\cdots\right) t^{2}+t^{3} \\
= & {\left[\left(a_{00}+a_{01} x+a_{02} x^{2}+\cdots\right)+t\right]\left[\left(b_{00}+b_{01} x+b_{02} x^{2}+\cdots\right)\right.} \\
& \left.\quad+\left(b_{10}+b_{11} x+b_{12} x^{2}+\cdots\right) t+t^{2}\right]
\end{aligned}
$$




$$
\begin{aligned}
\Leftrightarrow & \left(r_{00}+r_{10} t+r_{20} t^{2}+t^{3}\right)+\left(r_{01}+r_{11} t+r_{21} t^{2}\right) x+\left(r_{02}+r_{12} t+r_{22} t^{2}\right) x^{2}+\cdots \\
= & {\left[\left(a_{00}+t\right)+a_{01} x+a_{02} x^{2}+\cdots\right]\left[\left(b_{00}+b_{10} t+t^{2}\right)+\left(b_{01}+b_{11} t\right) x\right.} \\
& \left.+\left(b_{02}+b_{12} t\right) x^{2}+\cdots\right]
\end{aligned}
$$

$\Leftrightarrow$ the following equation system has a solution:

$$
\begin{array}{ll}
\left(P_{0}\right): & \left(a_{00}+t\right)\left(b_{00}+b_{10} t+t^{2}\right)=r_{00}+r_{10} t+r_{20} t^{2}+t^{3} \\
\left(P_{n}\right)(n \in \mathbb{N}): & a_{0 n}\left(b_{00}+b_{10} t+t^{2}\right)+a_{0, n-1}\left(b_{01}+b_{11} t\right)+\cdots+a_{01}\left(b_{0, n-1}+b_{1, n-1} t\right) \\
& +\left(a_{00}+t\right)\left(b_{0 n}+b_{1 n} t\right)=r_{0 n}+r_{1 n} t+r_{2 n} t^{2}
\end{array}
$$

$\left(P_{0}\right)$ holds by assumption of $h_{0}(t)$ and $h_{1}(t)$. Inductively, assume $\left(P_{n}\right), n=0,1, \ldots$, $k-1$, have solutions, and we prove $\left(P_{k}\right)$ is solvable.

$$
\begin{aligned}
\left(P_{k}\right): \quad & a_{0 k}\left(b_{00}+b_{10} t+t^{2}\right)+a_{0, k-1}\left(b_{01}+b_{11} t\right)+\cdots+a_{01}\left(b_{0, k-1}+b_{1, k-1} t\right) \\
& +\left(a_{00}+t\right)\left(b_{0 k}+b_{1 k} t\right)=r_{0 k}+r_{1 k} t+r_{2 k} t^{2} .
\end{aligned}
$$

Since the coefficients of $t$ in $a_{0, k-1}\left(b_{01}+b_{11} t\right)+\cdots+a_{01}\left(b_{0, k-1}+b_{1, k-1} t\right)$ are all known by induction hypothesis, $\left(P_{k}\right)$ is transformed into

$$
\begin{aligned}
(* *): & a_{0 k}\left(b_{00}+b_{10} t+t^{2}\right)+\left(a_{00}+t\right)\left(b_{0 k}+b_{1 k} t\right) \\
& =-\left[a_{0, k-1}\left(b_{01}+b_{11} t\right)+\cdots+a_{01}\left(b_{0, k-1}+b_{1, k-1} t\right)\right]+r_{0 k}+r_{1 k} t+r_{2 k} t^{2}
\end{aligned}
$$

with $a_{0 k}$ and $b_{0 k}, b_{1 k}$ being variables. Transfer $(* *)$ into linear equation system:

$$
\left\{\begin{array}{l}
a_{0 k} b_{00}+a_{00} b_{0 k}=r_{0 k}^{\prime} \\
a_{0 k} b_{10}+a_{00} b_{1 k}+b_{0 k}=r_{1 k}^{\prime} \\
a_{0 k}+b_{1 k}=r_{2 k}^{\prime}
\end{array}\right.
$$

with $r_{i k}^{\prime}, i=0,1,2$, being the coefficients of the polynomial on the right hand side of the equation (**). Define matrices $A, X, B$ as

$$
A=\left[\begin{array}{ccc}
b_{00} & a_{00} & 0 \\
b_{10} & 1 & a_{00} \\
1 & 0 & 1
\end{array}\right], X=\left[\begin{array}{c}
a_{0 k} \\
b_{0 k} \\
b_{1 k}
\end{array}\right], B=\left[\begin{array}{c}
r_{0 k}^{\prime} \\
r_{1 k}^{\prime} \\
r_{2 k}^{\prime}
\end{array}\right] .
$$

Then the above equation can be transformed into the matrix equation $A X=B$. $h^{\prime}(t)=h_{0}^{\prime}(t) h_{1}^{\prime}(t)$ is an $S R C$ factorization with $h^{\prime}(0)=h_{0}^{\prime}(0) h_{1}^{\prime}(0) \in J(R), h^{\prime}(1)=$ $h_{0}^{\prime}(1) h_{1}^{\prime}(1) \in J(R)$ and $h_{0}^{\prime}(0) \in U(R), h_{1}^{\prime}(1) \in U(R)$. So $a_{00} \in U(R), b_{00} \in J(R), b_{10}+$ $1 \in U(R)$ and $a_{00}+1 \in J(R)$. Hence $\operatorname{det} A=b_{00}+\left(a_{00}\right)^{2}-a_{00} b_{10}=b_{00}+a_{00}\left(a_{00}+\right.$ $1)-a_{00}\left(1+b_{10}\right) \in U(R)$. So the matrix equation is solvable and then so is equation $(* *)$ and by induction $\left(P_{n}\right)$ is solvable.

From case (a) and case (b), we know there exist $h_{0}(t)$ and $h_{1}(t)$ such that $h(t)=h_{0}(t) h_{1}(t) \quad$ and $\quad h_{0}(0) \in U(R[[x]]), \quad h_{1}(1) \in U(R[[x]])$. Now $\eta_{J(R[x]])}^{\prime}\left(h_{0}(t)\right)=$ $\left(\bar{\theta}^{\prime}\right)^{-1} \eta_{J(R)}^{\prime} \theta^{\prime}\left(h_{0}(t)\right)$ and $\eta_{J(R[[x])}^{\prime}\left(h_{1}(t)\right)=\left(\bar{\theta}^{\prime}\right)^{-1} \eta_{J(R)}^{\prime} \theta^{\prime}\left(h_{1}(t)\right)$. Since $\operatorname{gcd}\left(\eta_{J(R)}^{\prime} \theta^{\prime}\left(h_{0}(t)\right)\right.$, $\left.\eta_{J(R)}^{\prime} \theta^{\prime}\left(h_{1}(t)\right)\right)=1$, we $\operatorname{get} \operatorname{gcd}\left(\left(\bar{\theta}^{\prime}\right)^{-1} \eta_{J(R)}^{\prime} \theta^{\prime}\left(h_{0}(t)\right), \quad\left(\bar{\theta}^{\prime}\right)^{-1} \eta_{J(R)}^{\prime} \theta^{\prime}\left(h_{1}(t)\right)\right)=1$, i.e., $\operatorname{gcd}\left(\eta_{J(R[[x]])}^{\prime}\left(h_{0}(t)\right), \eta_{J(R[[x]])}^{\prime}\left(h_{1}(t)\right)\right)=1$. So $h(t)$ has an $S R C$ factorization, i.e., $R[[x]]$ is a $3-S R C$ ring.

$(2) \Rightarrow(1)$. By Lemma $2.2, \mathbb{M}_{3}(R[[x]])$ is strongly clean. So its image $\mathbb{M}_{3}(R)$ is also strongly clean. Again by Lemma $2.2, R$ is $3-S R C$.

We sketch proof of the following theorem; the proof is similar to that of Theorem 2.3. 
THEOREM 2.4. Let $R$ be a commutative local ring. Then the following are equivalent:

(1) $R$ is a 4-SRC ring.

(2) $R[[x]]$ is a $4-S R C$ ring.

Proof. $(1) \Rightarrow(2)$ : Define ring homomorphisms similar to that in Theorem 2.3. Then the following diagrams commute.
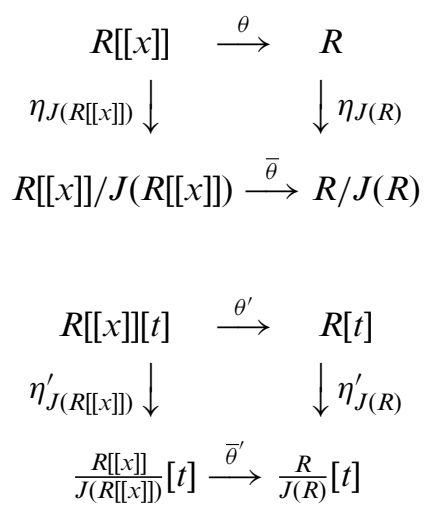

Let $h(t)=f_{0}+f_{1} t+f_{2} t^{2}+f_{3} t^{3}+t^{4} \in R[[x]][t]$ with $f_{i}=r_{i 0}+r_{i 1} x+r_{i 2} x^{2}+\cdots \epsilon$ $R[[x]], i=0,1,2,3$.

I: If $h(0) \in U(R)$, or $h(1) \in U(R)$, then as in Theorem 2.3, $h(t)$ has an $S R C$ factorization. II: If $h(0)=f_{0} \in J(R[[x]]), h(1)=f_{0}+f_{1}+f_{2}+f_{3}+1 \in J(R[[x]])$, i.e., $r_{00} \in J(R)$, and $r_{00}+r_{10}+r_{20}+r_{30}+1 \in J(R)$, we prove $h(t)$ has an $S R C$ factorization.

Let $h^{\prime}(t)=\theta^{\prime}(h(t))$. Then $h^{\prime}(t)=r_{00}+r_{10} t+r_{20} t^{2}+r_{30} t^{3}+t^{4}, h^{\prime}(0)=r_{00} \in J(R)$ and $h^{\prime}(1)=r_{00}+r_{10}+r_{20}+r_{30}+1 \in J(R)$. Since $R$ is a $4-S R C$ ring, there exist the following three cases

$$
\text { (a) }\left\{\begin{array}{l}
h_{0}^{\prime}(t)=a_{00}+a_{10} t+a_{20} t^{2}+t^{3} \\
h_{1}^{\prime}(t)=b_{00}+t
\end{array}\right.
$$

or

(b) $\left\{\begin{array}{l}h_{0}^{\prime}(t)=a_{00}+a_{10} t+t^{2} \\ h_{1}^{\prime}(t)=b_{00}+b_{10} t+t^{2}\end{array}\right.$

or

$$
\text { (c) }\left\{\begin{array}{l}
h_{0}^{\prime}(t)=a_{00}+t \\
h_{1}^{\prime}(t)=b_{00}+b_{10} t+b_{20} t^{2}+t^{3}
\end{array}\right.
$$

such that $h_{0}^{\prime}(0) \in U(R), h_{1}^{\prime}(1) \in U(R), \operatorname{gcd}\left(\eta_{J(R)}^{\prime}\left(h_{0}(t)\right), \eta_{J(R)}^{\prime}\left(h_{1}(t)\right)\right)=1$ and $h^{\prime}(t)=$ $h_{0}^{\prime}(t) h_{1}^{\prime}(t)$.

Case (a):

$$
\left\{\begin{array}{l}
h_{0}^{\prime}(t)=a_{00}+a_{10} t+a_{20} t^{2}+t^{3} \\
h_{1}^{\prime}(t)=b_{00}+t .
\end{array}\right.
$$


Similar to case (a) in Theorem 2.3, we prove the following matrix $A$ is invertible.

$$
A=\left[\begin{array}{cccc}
b_{00} & 0 & 0 & a_{00} \\
1 & b_{00} & 0 & a_{10} \\
0 & 1 & b_{00} & a_{20} \\
0 & 0 & 1 & 1
\end{array}\right]
$$

By $h_{0}^{\prime}(0) \in U(R), h_{1}^{\prime}(1) \in U(R), h^{\prime}(0)=h_{0}^{\prime}(0) h_{1}^{\prime}(0) \in J(R) h^{\prime}(1)=h_{0}^{\prime}(1) h_{1}^{\prime}(1) \in J(R)$, we get $a_{00} \in U(R)$ and $b_{00} \in J(R)$. So $\operatorname{det} A=b_{00}\left(b_{00}^{2}-a_{10}-b_{00} a_{20}\right)-a_{00} \in U(R)$.

Case (b):

$$
\left\{\begin{array}{l}
h_{0}^{\prime}(t)=a_{00}+a_{10} t+t^{2} \\
h_{1}^{\prime}(t)=b_{00}+b_{10} t+t^{2}
\end{array}\right.
$$

As in case (a), we prove $A$ is invertible where

$$
A=\left[\begin{array}{cccc}
b_{00} & 0 & a_{00} & 0 \\
b_{10} & b_{00} & a_{10} & a_{00} \\
1 & b_{10} & 1 & a_{10} \\
0 & 1 & 0 & 1
\end{array}\right]
$$

By $h_{0}^{\prime}(0) \in U(R), h_{1}^{\prime}(1) \in U(R), h^{\prime}(0)=h_{0}^{\prime}(0) h_{1}^{\prime}(0) \in J(R) h^{\prime}(1)=h_{0}^{\prime}(1) h_{1}^{\prime}(1) \in J(R)$ and $\operatorname{gcd}\left(\overline{h_{0}^{\prime}}(t), \overline{h_{1}^{\prime}}(t)\right)=1$, we get $b_{00} \in J(R), a_{00} \in U(R), 1+b_{10} \in U(R)$, and $b_{10}-$ $a_{10}-1 \in U(R)$ and $a_{00}=-a_{10}-1+j$ for some $j \in J(R)$. So

$$
\begin{aligned}
\operatorname{det} A & =b_{00}\left(b_{00}+a_{10}^{2}-b_{00}-a_{10} b_{00}\right)-a_{00}\left(b_{10}^{2}+a_{00}-b_{00}-a_{10} b_{10}\right) \\
& =b_{00}\left(b_{00}+a_{10}^{2}-b_{00}-a_{10} b_{00}\right)-a_{00}\left[\left(b_{10}+1\right)\left(b_{10}-a_{10}-1\right)+j-b_{00}\right] \in U(R) .
\end{aligned}
$$

Case (c):

$$
\left\{\begin{array}{l}
h_{0}^{\prime}(t)=a_{00}+t \\
h_{1}^{\prime}(t)=b_{00}+b_{10} t+b_{20} t^{2}+t^{3}
\end{array}\right.
$$

As before,

$$
A=\left[\begin{array}{cccc}
b_{00} & a_{00} & 0 & 0 \\
b_{10} & 1 & a_{00} & 0 \\
b_{20} & 0 & 1 & a_{00} \\
1 & 0 & 0 & 1
\end{array}\right]
$$

By $h_{0}^{\prime}(0) \in U(R), h_{1}^{\prime}(1) \in U(R), h^{\prime}(0)=h_{0}^{\prime}(0) h_{1}^{\prime}(0) \in J(R), h^{\prime}(1)=h_{0}^{\prime}(1) h_{1}^{\prime}(1) \in J(R)$, we get $a_{00} \in U(R), \quad b_{00} \in J(R), \quad 1+a_{00} \in J(R), \quad b_{00}+b_{10}+b_{20}+1 \in U(R)$ and $a_{00}-b_{10}-b_{20} \in U(R)$. So $\operatorname{det} A=b_{00}-a_{00}^{2}\left(a_{00}-b_{10}-b_{20}\right)-a_{00}\left(a_{00}+1\right) b_{10} \in U(R)$. Again repeat the last part of Theorem 2.3; $h(t)$ has an $S R C$ factorization, i.e., $R[[x]]$ is a $4-S R C$ ring.

$(2) \Rightarrow(1)$ is similar to the above theorem.

Before giving the main theorem, we need more lemmas.

LEMMA 2.5. Let $R$ be a ring. Then $\frac{R[x]}{\left(x^{k}\right)} \cong \frac{R[[x]]}{\left(x^{k}\right)}$. 
Proof. Define $\theta: R[[x]] \rightarrow \frac{R[x]}{\left(x^{k}\right)}$ by $\theta\left(\sum_{i \geq 0} r_{i} x^{i}\right)=r_{0}+r_{1} \bar{x}+\cdots+r_{k} \bar{x}^{k}$ where $\bar{x}=$ $x+\left(x^{k}\right)$. Then $\theta$ is a ring epimorphism with $\operatorname{Ker} \theta=\left(x^{k}\right)$. So $\frac{R[x]}{\left(x^{k}\right)} \cong \frac{R[x]]}{\left(x^{k}\right)}$.

LEMMA 2.6. Let $R$ be a local ring. Then $\frac{R\left[x_{1}, x_{2}, \ldots, x_{k}\right]}{\left(x_{1}^{n_{1}}, x_{2}^{n_{2}}, \ldots, x_{k}^{n_{k}}\right)}$ is a local ring.

Proof. Let $I=\frac{\left(x_{1}, x_{2}, \ldots, x_{k}\right)}{\left(x_{1}^{n_{1}}, x_{2}^{n_{2}}, \ldots, x_{k}^{n_{k}}\right)}$. Then $I$ a nilpotent ideal. Define

$$
\theta: R\left[x_{1}, x_{2}, \ldots, x_{k}\right] \rightarrow \frac{R\left[x_{1}, x_{2}, \ldots, x_{k}\right]}{\left(x_{1}^{n_{1}}, x_{2}^{n_{2}}, \ldots, x_{k}^{n_{k}}\right)}
$$

to be the natural ring epimorphism. Then by [1, Corollary 15.8$]$, we have

$$
\frac{J(R)+\left(x_{1}^{n_{1}}, x_{2}^{n_{2}}, \ldots, x_{k}^{n_{k}}\right)}{\left(x_{1}^{n_{1}}, x_{2}^{n_{2}}, \ldots, x_{k}^{n_{k}}\right)} \subseteq J\left(\frac{R\left[x_{1}, x_{2}, \ldots, x_{k}\right]}{\left(x_{1}^{n_{1}}, x_{2}^{n_{2}}, \ldots, x_{k}^{n_{k}}\right)}\right) .
$$

So

$$
\frac{J(R)+\left(x_{1}, x_{2}, \ldots, x_{k}\right)}{\left(x_{1}^{n_{1}}, x_{2}^{n_{2}}, \ldots, x_{k}^{n_{k}}\right)} \subseteq J\left(\frac{R\left[x_{1}, x_{2}, \ldots, x_{k}\right]}{\left(x_{1}^{n_{1}}, x_{2}^{n_{2}}, \ldots, x_{k}^{n_{k}}\right)}\right) .
$$

Hence,

$$
\frac{\frac{R\left[x_{1}, x_{2}, \ldots, x_{k}\right]}{\left(x_{1}^{n_{1}}, x_{2}^{n_{2}}, \ldots, x_{k}^{n_{k}}\right)}}{\frac{J(R)+\left(x_{1}, x_{2}, \ldots, x_{k}\right)}{\left(x_{1}^{n_{1}}, x_{2}^{n_{2}}, \ldots, x_{k}^{n_{k}}\right)}} \cong \frac{R\left[x_{1}, x_{2}, \ldots, x_{k}\right]}{J(R)+\left(x_{1}, x_{2}, \ldots, x_{k}\right)} \cong \frac{R}{J(R)}
$$

is a division ring. And $\frac{R\left[x_{1}, x_{2}, \ldots, x_{k}\right]}{\left(x_{1}^{n_{1}}, x_{2}, \ldots, x_{k}\right)}$ is a local ring.

LEMMA 2.7. Let $R$ be a ring. Then $\frac{R\left[x_{1}, x_{2}, \ldots, x_{k}\right]}{\left(x_{1}^{n_{1}}, x_{2}^{n_{2}}, \ldots, x_{k}^{k_{k}}\right)} \cong \frac{\left.R\left[x_{1}, x_{2}, \ldots, x_{k}\right]\right]}{\left(x_{1}^{n_{1}}, x_{2}^{n_{2}}, \ldots, x_{k}^{k_{k}}\right)}$.

Proof. The proof is similar to Lemma 2.5.

Definition 2.8. $R \propto R=\left\{\left(\begin{array}{cc}a & b \\ 0 & a\end{array}\right): a, b \in R\right\}$ is called the trivial extension of $R$.

LEMMA 2.9. Let $R$ be a ring. Then $R \propto R \cong \frac{R[x]}{\left(x^{2}\right)}$.

Proof. Define $\theta: R \propto R \rightarrow \frac{R[x]}{\left(x^{2}\right)}$ by $\theta\left(\left(\begin{array}{ll}a & b \\ 0 & a\end{array}\right)\right)=a+b \bar{x}$ with $\bar{x}=x+\left(x^{2}\right)$. It is easy to verify that $\theta$ is an isomorphism.

The following is the main result.

THEOREM 2.10. Let $R$ be a commutative local ring, and let $n=3,4$, and $m, k, s \in \mathbb{N}$. Then the following are equivalent:

1. $\mathbb{M}_{n}(R)$ is strongly clean.

2. $\mathbb{M}_{n}(R[[x]])$ is strongly clean.

3. $\mathbb{M}_{n}\left(\frac{R[x]}{\left(x^{m}\right)}\right)$ is strongly clean.

4. $\mathbb{M}_{n}\left(R\left[\left[x_{1}, x_{2}, \ldots, x_{k}\right]\right]\right)$ is strongly clean.

5. $\mathbb{M}_{n}\left(\frac{R\left[x_{1}, x_{2}, \ldots, x_{s}\right]}{\left(x_{1}^{n_{1}}, x_{2}^{n_{2}}, \ldots, x_{s}^{n_{s}}\right)}\right)$ is strongly clean.

6. $\mathbb{M}_{n}(R \propto R)$ is strongly clean. 
Proof. Note by the conditions, known results or above lemmas, $R, R[[x]], \frac{R[x]}{\left(x^{m}\right)}$, $R\left[\left[x_{1}, x_{2}, \ldots, x_{k}\right]\right], \frac{R\left[x_{1}, x_{2}, \ldots, x_{s}\right]}{\left(x_{1}^{n_{1}}, x_{2}^{n_{2}}, \ldots, x_{s}^{n_{s}}\right)}$, and $R \propto R$ are all commutative local.

(1) $\Leftrightarrow(2)$. By Theorem 2.3, Theorem 2.4 and Lemma 2.2.

$(2) \Rightarrow(3) \Rightarrow(1) \cdot \mathbb{M}_{n}(R)$ is the image of $\mathbb{M}_{n}\left(\frac{R[x]}{\left(x^{m}\right)}\right)$ and $\mathbb{M}_{n}\left(\frac{R[x]}{\left(x^{m}\right)}\right)$ is the image of $\mathbb{M}_{n}(R[[x]])$ by Lemma 2.5 .

(2) $\Leftrightarrow(4)$. By mathematical induction, Lemma 2.2, Theorem 2.3 and Theorem 2.4.

$(4) \Rightarrow(5) \Rightarrow(1)$. This is similar to $(2) \Rightarrow(3) \Rightarrow(1)$ because by Lemma 2.7 , $\frac{R\left[x_{1}, x_{2}, \ldots, x_{k}\right]}{\left(x_{1}^{n_{1}}, x_{2}^{n_{2}}, \ldots, x_{k}^{n_{k}}\right)} \cong \frac{R\left[\left[x_{1}, x_{2}, \ldots, x_{k}\right]\right]}{\left(x_{1}^{n_{1}}, x_{2}^{n_{2}}, \ldots, x_{k}^{n_{k}}\right)}$.

$(1) \Leftrightarrow(6)$. (1) $\Leftrightarrow(3)$ for any $m \in \mathbb{N}$. So (6) is a special case of (3) if we let $m=2$.

The following is an application of Theorem 2.10.

A commutative local ring $R$ is called Henselian if $R[x]$ satisfies Hensel's lemma $[3,9]$, i.e., for any monic polynomial $f(x) \in R[x]$, if $\bar{f}(x)=\bar{g}(x) \bar{h}(x)$ with $\bar{g}(x), \bar{h}(x) \in$ $\frac{R}{J(R)}[x]$ monic and coprime, then there exist monic polynomials $g(x)$ and $h(x)$ in $R[x]$ such that $f(x)=g(x) h(x), \eta_{R}^{\prime}(g(x))=\bar{g}(x)$, and $\eta_{R}^{\prime}(h(x))=\bar{h}(x)$.

LEMMA 2.11. [4] If $R$ is Henselian, then $R$ is an SRC ring.

COROLlary 2.12. For any prime number $p$, let $\widehat{\mathbb{Z}}_{p}$ be the ring of $p$-adic integers, and let $n=3,4$ and $m, k, s \in \mathbb{N}$. Then $\mathbb{M}_{n}\left(\widehat{\mathbb{Z}}_{p}[[x]]\right), \mathbb{M}_{n}\left(\frac{\widehat{\mathbb{Z}}_{p}[x]}{\left(x^{k}\right)}\right), \mathbb{M}_{n}\left(\widehat{\mathbb{Z}}_{p}\left[\left[x_{1}, x_{2}, \ldots, x_{m}\right]\right]\right)$, $\mathbb{M}_{n}\left(\frac{\widehat{\mathbb{Z}}_{p}\left[x_{1}, x_{2}, \ldots, x_{s}\right]}{\left(x_{1}^{n_{1}}, x_{2}^{n_{2}}, \ldots, x_{s}^{n_{s}}\right)}\right)$, and $\mathbb{M}_{n}\left(\widehat{\mathbb{Z}}_{p} \propto \widehat{\mathbb{Z}}_{p}\right)$ are all strongly clean.

Proof. By [6, Theorem 7.18], $\widehat{\mathbb{Z}}_{p}$ is Henselian. (For definitions and properties of $\widehat{\mathbb{Z}}_{p}$ and Hensel's lemma, see [7].) So by Theorem 2.10, Lemma 2.2 and Lemma 2.11, the corollary holds.

Corollary 2.13. Let $F$ be a field, and let $n=3,4$ and $m, k, s \in \mathbb{N}$. Then $\mathbb{M}_{n}(F[[x]])$, $\mathbb{M}_{n}\left(\frac{F[x]}{\left(x^{k}\right)}\right), \mathbb{M}_{n}\left(F\left[\left[x_{1}, x_{2}, \ldots, x_{m}\right]\right]\right), \mathbb{M}_{n}\left(\frac{F\left[x_{1}, x_{2}, \ldots, x_{s}\right]}{\left(x_{1}^{n_{1}}, x_{2}^{n_{2}}, \ldots, x_{s}^{n_{s}}\right)}\right)$ and $\mathbb{M}_{n}(F \propto F)$ are strongly clean.

Proof. By [2, p. 115, ex. 9], $F$ is Henselian. So again by Theorem 2.10, Lemma 2.2 and Lemma 2.11, the corollary holds.

ACKNOWLEDGEMENTS. The authors are grateful to the referee who has carefully read the article and made many valuable comments for the revision. They also thank Dr. A. Diesl for his comments.

\section{REFERENCES}

1. F. Anderson and K. Fuller, Rings and categories of modules, second edition (SpringerVerlag, 1992).

2. M. F. Atiyah and I. G. MacDonald, Introduction to commutative algebra (AddisonWesley, 1969).

3. G. Azumaya, On maximally central algebras, Nagoya Math. J. 2(1950), 119-150.

4. G. Boroozh, A. Diesl and T. Dorsey, Strongly clean matrix rings over commutative local rings, preprint, 2005.

5. J. Chen, X. Yang and Y. Zhou, When is the $2 \times 2$ matrix ring over a commutative local ring strongly clean? J. Algebra 301(2006), 280-293.

6. D. Eisenbud, Commutative algebra with a view toward algebraic geometry (SpringerVerlag, 1994).

7. F. Q. Gouvêa, p-Adic numbers. An introduction. (Springer-Verlag, 1993). 2595.

8. J. Han and W. K. Nicholson, Extensions of clean rings, Comm. Algebra 29(2001), 2589- 
9. M. Nagata, On the theory of Henselian rings, Nagoya Math. J. 5(1953), 45-57.

10. W. K. Nicholson, Lifting idempotents and exchange rings, Trans. Amer. Math. Soc. 229(1977), 269-278.

11. W. K. Nicholson, Strongly clean rings and Fitting's lemma, Comm. Algebra 27(1999), $3583-3592$.

12. E. Sánchez Campos, On strongly clean rings. Unpublished.

13. Z. Wang and J. Chen, On two open problems about strongly clean rings, Bull. Austral. Math. Soc. 70(2004), 279-282. 